\title{
A Universidade Colaborando na Construção de um Projeto de Promoção da Saúde: Relato de Experiência de um Grupo de Alunos de Medicina da Unicamp, Campinas, SP, Brasil
}

\author{
The University Collaborating in the \\ Construction of a Health Promotion Project: \\ Report on an Experience of a Group of Medical \\ Students at Unicamp, Campinas, SP, Brazil
}

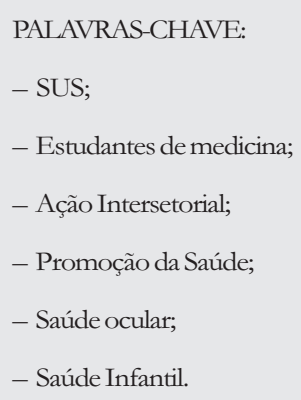

Ana Maria Girotti Sperandio ${ }^{1}$

Thiago Ferreira de Souza ${ }^{1}$ Edson Breno ${ }^{1}$

Leandro César Mendes ${ }^{1}$

Araceli Regina S. Pereira ${ }^{1}$

Alexandre Cason Machado ${ }^{1}$ Alice Jordão de Toledo Mazon ${ }^{1}$

Natália Reis Verderost ${ }^{1}$

Marcos Teófilo Galasso ${ }^{1}$ Letícia Pisoni Zanaga $^{1}$ Wilmar Azal Neto ${ }^{1}$ Edison Bueno ${ }^{1}$

\section{RESUMO}

Este trabalho foi realizado com o objetivo de oferecer aos estudantes de graduação em Medicina da Universidade Estadual de Campinas (Unicamp) a vivência de ações de saúde pública, segundo princípios do Sistema Único de Saúde (SUS), por meio da elaboração de um projeto coletivo de promoção da saúde ocular de crianças de 0 a 7 anos. Para isto, foi aplicado um questionário para levantar as necessidades em relação à saúde ocular em uma amostra da população usuária do Centro de Saúde Jardim Santa Mônica em Campinas (SP), em 2003. Nele, percebeu-se a falta de informações sobre cuidados básicos com os olhos e de recursos e qualificação profissional para diagnóstico e tratamento, bem como o desconhecimento dos direitos à saúde e a ausência de medidas preventivas, principalmente para crianças. Foi desenvolvido, então, um projeto de ação e aprendizado com alunos, professores, agentes comunitários de saúde e auxiliares de uma creche do bairro, formando uma equipe de trabalho. Foram realizadas oficinas com essa equipe, na perspectiva da promoção e proteção da saúde ocular. Para a sustentabilidade do projeto, o planejamento previu a criação de um banco de óculos para a doação de armações e garantia da confecção para as pessoas com dificuldades financeiras. A experiência da construção e da aplicação do projeto permitiu o conhecimento, na prática, da organização dos serviços e a dinâmica do sistema de saúde, inclusive para compreender alguns limites e sugerir políticas públicas de saúde ocular que correspondam às necessidades da população, apontando-se o papel do médico no desencadeamento de um trabalho coletivo de compartilhamento de saberes e responsabilidades. 


\begin{abstract}
The goal of this project was to promote eye health and provide eye care to children from 0 to 7 years of age and to offer to medical students of Unicamp the possibility of participating in practice in a public health action carried out according to the principles of the Brazilian Unified Health System (SUS - Sistema Único de Saúde). In 2003, a questionnaire was applied to a sample of users of the Jardim Santa Mônica Health Care Center in Campinas, SP. Analysis of the data there obtained revealed some deficiencies in the promotion of eye health such as lack of information about basic eye care, lack of resources for treatment, lack of information about the right to care by the Unified Health System and the absence of any preventive measures, mainly for children. Thus, a project was developed offering workshops for students, teachers, community health agents and personnel from a neighborhood nursery-school to enable them to act as multipliers of the obtained knowledge and to initiate a process of awareness building. A spectacle bank was created in order to grant the sustainability of the project, offering the confection of eye glasses with frames donated by the population and lenses offered by the city government and some optic stores to needed persons. Through this experience the medical students could obtain some practical knowledge about the organization of health services and the dynamics of the health system, enabling them to understand some limitations and to suggest public eye health policies meeting the needs of the population. A closer study of this project shows not only how important this kind of action is for the most needed segments of society but also the role a doctor can play as someone able to convince people to go for their rights.
\end{abstract}

A melhor maneira que nós temos de pensar mais ou menos certo é pensar a prática e saber que esta prática não é individual, mas social.

Paulo Freire

\section{INTRODUÇÃO}

O Sistema Único de Saúde (SUS), regulamentado em 1990, baseia-se nos princípios de integralidade, eqüidade e universalidade, entre outros, e nas ações de promoção da saúde, na prevenção de agravos à saúde, tratando, reabilitando, colaborando na redução das desigualdades e cuidando de cada pessoa segundo suas necessidades, colocando a saúde como um direito assegurado pelo Estado e provendo o acesso às ações e aos serviços independentemente de sexo, cor, raça, renda, ocupação ou outras características pessoais ou sociais ${ }^{1}$.
Deve haver garantia de atenção à saúde por parte do sistema a todo e qualquer cidadão. Com a universalidade, o indivíduo passa a ter direito de acesso a todos os serviços públicos de saúde, assim como àqueles contratados pelo poder público. Saúde é direito de cidadania e dever de provimento dos governos municipal, estadual e federal.

Sabe-se que, para a construção de um plano de ação de promoção da saúde, são necessários o compromisso do governo local e a participação popular nas diferentes etapas do processo: no diagnóstico local, na execução e na avaliação, sendo que esta última deve ser constante ${ }^{2}$. A promoção da saúde consiste em "proporcionar aos povos os meios necessários para melhorar a sua saúde e exercer um maior controle sobre ela", segundo a Carta de Ottawa, $1986^{3}$.

Com o objetivo de fortalecer e criar espaços para a construção de políticas públicas saudáveis, o Ministério da Saúde, no documento da política de promoção da saúde, expressa que deve ser uma:

estratégia de articulação transversal na qual se confere visibilidade aos fatores que colocam a saúde da população em risco e às diferenças entre necessidades, territórios e culturas presentes no nosso país, visando à criação de mecanismos que reduzam as situações de vulnerabilidade, defendam radicalmente a eqüidade e incorporem a participação e o controle sociais na gestão das políticas públicas... ${ }^{4}$

ampliando, dessa forma, a atuação de diferentes setores na busca da elaboração de projetos que alcancem melhorias na qualidade de vida.

Por outro lado, a mudança curricular nos cursos de Medicina, nos últimos cinco anos, criou um espaço de possibilidade de aprendizado com alunos a partir do primeiro ano de graduação de Medicina para desenvolver projetos que mostrem a importância e a valorização da eqüidade, a qualidade, a eficiência e a relevância do trabalho em saúde e, ainda, que o sucesso depende também da integração com outras áreas do saber.

Publicações na área de educação médica fazem referências a programas de formação médica que desvinculam a teoria da prática, dissociam os ciclos básico e clínico, e enfatizam especialidades médicas ligadas ao uso intenso de tecnologias. Isto pode contribuir para a formação de profissionais médicos com uma prática impessoal, descontextualizada, de forma a não contemplar as necessidades daqueles que procuram o serviço de saúde 5 .

Assim, o que se propõe como alternativa é o desenvolvimento de um processo de ensino centrado no estudante e em 
seu aprendizado, respeitando seus conhecimentos e vivências anteriores, e centrado também na relação entre professores e alunos ${ }^{6}$.

Um dos objetivos principais desta mudança é formar um médico generalista, humanista, crítico e reflexivo. Capacitado a atuar, respeitando princípios éticos, no processo de saúde-doença em seus diferentes níveis de atenção, com ações de promoção, prevenção, recuperação e reabilitação à saúde, na perspectiva da integralidade da assistência, com senso de responsabilidade social e compromisso com a cidadania ${ }^{6}$.

Para ser generalista, este profissional deve entender um pouco das diferentes áreas da saúde, principalmente os conceitos e ações básicas de saúde.

Desta forma, entende-se que a apropriação dos conhecimentos básicos deva incluir os da saúde ocular, fazendo parte do processo educativo na direção da promoção da saúde.

A falta de conhecimentos das pessoas em relação aos seus direitos à saúde se amplia para as outras áreas e, conforme registrado em alguns trabalhos científicos, principalmente em áreas específicas. Sperandio7, em 1999, apontou que grande parte dos próprios enfermeiros e pediatras que trabalhavam nos centros de saúde de Campinas naquele ano desconheciam as ações básicas de saúde ocular e recomendou que deveriam ser capacitados para aplicação das ações de saúde ocular em respeito à lei e ao direito de acesso às ações básicas de saúde. Ele refere ainda que os objetivos de programas educativos em saúde ocular deveriam contemplar não apenas acesso a conhecimentos sobre a área, mas também mudanças de atitudes e de práticas da população, dos serviços públicos, privados e filantrópicos, e também a formação dos alunos, principalmente dos cursos de Medicina e Enfermagem.

Aproveitando o espaço de formação criado a partir da mudança curricular, este trabalho teve como objetivo descrever a experiência da construção coletiva de um projeto intersetorial na perspectiva da promoção da saúde, especificamente na área de saúde ocular, desenvolvido por alguns discentes de primeiro ano de Medicina da Universidade Estadual de Campinas (Unicamp), em 2003, sob a coordenação da pesquisadora Ana Maria Girotti Sperandio, do Departamento de Medicina Preventiva e Social, no Bairro Santa Mônica, em Campinas (SP).

O artigo abordará a situação, em linhas gerais, da saúde ocular no Brasil; em seguida, os objetivos, metodologias, resultados e conclusões do projeto desenvolvido pelos alunos de primeiro ano em 2003.

\section{Saúde ocular: dados ainda em construção}

Situando a saúde ocular no contexto da saúde pública, dados preliminares do IBGE do Censo de 2000 indicam que a primeira causa de deficiência entre 24,5 milhões de deficientes brasileiros é a visual, representando $48,1 \%$ do total, o que revela um aumento na busca do serviço de saúde ocular para resolver os problemas sentidos. Cerca de $20 \%$ das crianças em idade escolar apresentam algum problema ocular, e aproximadamente $95 \%$ deles poderiam ser evitados se fossem aplicadas ações de promoção e prevenção por meio do processo educativo ${ }^{8}$. Kara-José ${ }^{\text {, }}$ um dos autores de referência nacional de trabalhos direcionados à saúde pública, destacou a importância de desenvolver ações preventivas com crianças.

A Organização Mundial da Saúde esclarece que cerca de $70 \%$ dos casos de cegueira mundial poderiam ser evitados se utilizássemos técnicas simples, de baixo custo, como a de aplicação de testes para avaliar a quantidade de visão10,11,12.

Atualmente, a situação da área corresponde, em sua grande maioria, a iniciativas de triagens visuais em escolares e programas para os idosos, com soluções curativas diante dos problemas detectados. É imprescindível deixar claro que não se pretende priorizar uma "parte do corpo", os olhos, já que o indivíduo é uma totalidade, incluindo um corpo, sentimentos, emoções, valores, e que se desenvolve imerso em uma dada realidade social, econômica, política e cultural. Isto implica, portanto, encarar a saúde ocular como parte integrante da saúde, entendida em seu sentido amplo, como processo biológico-social, e vislumbrar as possibilidades de direcionar estratégias específicas na perspectiva da promoção da saúde e prevenção de problemas.

O processo de construção/reconstrução das práticas de saúde ocular busca a socialização de conhecimentos básicos da área em questão e, mediante um processo participativo de uma dada população, contribuir para o fortalecimento individual e coletivo, conduzindo à construção de práticas transformadoras do quadro atual. Desta forma, abrem-se oportunidades para a construção de novas políticas públicas em saúde ocular, envolvendo a articulação de conhecimentos de distintas disciplinas e o ato de colocá-los ao alcance de outros cidadãos e, com eles, construir novos conhecimentos e novas práticas.

Esta deveria ser a concepção norteadora na construção de projetos de promoção da saúde ocular. O pressuposto de situar desse modo a saúde ocular indica que uma boa condição de saúde ocular faz parte de uma boa condição de saúde. E esta, constituindo um direito do cidadão, permite entender que qualquer pessoa tem o direito de conhecer e preservar sua saúde ocular, cabendo ao Estado a atenção e os cuidados necessários para mantê-la. 


\section{METODOLOGIA}

\section{Projeto desenvolvido com os alunos de Medicina}

O projeto desenvolveu-se a partir da disciplina Ações de Saúde Pública, ministrada no primeiro ano de Medicina da Unicamp, em 2003, e que tem como proposta analisar a situação das comunidades de Campinas utilizando técnicas para diagnóstico e indicadores de saúde. O objetivo é capacitar os discentes para trabalhos com grupos de diferentes atores sociais por meio da aplicação de programas de prevenção e educação em saúde.

No desenvolvimento deste projeto, que inclui a construção coletiva de ações na direção da promoção da saúde, foi considerada a premissa do SUS. Para isto, foi eleita uma área - a saúde ocular - para a construção de ações estratégicas de promoção da saúde na linha da pesquisa-ação ${ }^{13}$.

As ações em Saúde Pública propostas neste projeto se pautaram nos eixos da promoção da saúde como referida no documento do Ministério da Saúde, que aponta a necessidade de articulação entre os diferentes setores para reduzirmos as desigualdades ${ }^{4}$.

O projeto foi elaborado com base nos princípios do SUS, em especial a universalidade de acesso aos serviços de saúde em todos os níveis de assistência; a integralidade de assistência, entendida como conjunto articulado e contínuo das ações e serviços preventivos e curativos, individuais e coletivos, exigidos para cada caso em todos os níveis de complexidade do sistema; preservação da autonomia das pessoas na defesa de sua integralidade física e moral; igualdade de assistência à saúde, sem preconceitos e privilégios de qualquer espécie; o direito à informação, das pessoas assistidas, sobre sua saúde e, ainda, a participação da comunidade - tudo isso definido na chamada Lei Orgânica da Saúde, n 8.080, de 19 de setembro de $1990{ }^{14}$.

Esse projeto teve como objetivos levantar as necessidades em saúde ocular da população do Jardim Santa Mônica; elaborar coletivamente um projeto a partir das necessidades levantadas, envolvendo outras instituições; a partir do projeto, promover a saúde ocular por meio da realização de diagnóstico e tratamento precoces, e prevenção de problemas visuais em crianças de 0 a 7 anos; desencadear uma ação completa que constituísse a vivência do princípio da integralidade, partindo do reconhecimento do problema e chegando à implantação de ações corretivas de curto, médio e longo prazo, buscando a multiplicação de conhecimentos dos diferentes atores sociais; caracterizar, junto à população-alvo, a saúde ocular como um direito e uma necessidade enquanto ação básica de saúde e forma de prevenção eficaz contra os princi- pais e mais prevalentes problemas visuais; vivenciar, por parte dos estudantes de graduação do primeiro ano de Medicina, uma experiência de realização de ações em saúde pública considerando, para isto, a análise da situação de um território (quanto à saúde ocular) e chegando a uma ação efetiva de intervenção.

Na primeira etapa, os estudantes do grupo envolvidos no projeto realizaram, por meio de um questionário, o reconhecimento do território (Bairro Jardim Santa Mônica, Campinas, SP) e o levantamento dos problemas de saúde, por intermédio de observações diretas e de dados fornecidos pelo Centro de Saúde, com o auxílio de agentes comunitários e moradores do bairro.

Na segunda etapa, com o auxílio do docente responsável, foi indicado um profissional para a implementação da proposta mais relevante segundo a análise do questionário aplicado. A partir das discussões entre docentes, discentes e representantes da equipe técnica acerca das necessidades de saúde da comunidade do Jardim Santa Mônica, foi decidido realizar uma ação na área de saúde ocular, considerando também a grande demanda nessa área.

Dessa forma, um novo questionário (Anexo) foi aplicado no Centro de Saúde, pelo qual se verificou o desconhecimento da população pesquisada a respeito de seus direitos à promoção e proteção de sua saúde visual, aos cuidados que devem ser tomados com os olhos e se já haviam realizado algum tipo de triagem visual (consulta, tratamento).

As respostas obtidas com o questionário confirmaram a suspeita levantada pelo grupo: a de que havia um déficit de conhecimentos em relação à saúde ocular e ao acesso às ações básicas de saúde ocular. Além disso, foi notada a ausência de ações básicas preventivas entre crianças de até sete anos, período crítico para a formação do sistema visual e durante o qual se pode evitar o desenvolvimento e a instalação de deficiências que após esse período se tornarão irreversíveis.

Identificadas as necessidades, iniciou-se a elaboração de um projeto de ação coletivo, voltado à promoção da saúde ocular e à prevenção precoce de problemas visuais em crianças dessa faixa etária, para solucionar as deficiências encontradas $^{15}$. O projeto de ação foi apresentado à direção da creche existente no bairro, que o aceitou, considerando sua importância para a promoção da saúde ocular. Assim, foi realizado um processo de capacitação por meio de oficinas com discentes da Unicamp, agentes comunitários de saúde e professores da creche para a aplicação dos testes de triagem visual. As oficinas foram oferecidas por profissionais especializados em Oftalmologia e Saúde Pública, para transmitir informações básicas, técnicas para a realização de testes de acuidade visual 
e informações sobre direitos e deveres do usuário do Sistema Único de Saúde no que diz respeito a ações básicas de saúde com as crianças.

Foi realizado, então, um processo de capacitação, por meio de oficinas oferecidas aos estudantes de Medicina, agentes de saúde e professores da creche, por profissionais especializados na área de Oftalmologia* e Ortóptica/Saúde Pública (a responsável pelo projeto). $\mathrm{O}$ material constava de apostilas e fotos de casos clínicos. Os conteúdos transmitidos foram: informações básicas acerca da anatomia do aparelho visual; conhecimento das principais doenças do aparelho visual, seus tratamentos e medidas de prevenção, assim como técnicas para realização de testes de acuidade visual; direitos e deveres do usuário do Sistema Único de Saúde no que diz respeito às ações básicas de saúde junto às crianças; técnicas de avaliação da acuidade visual.

O projeto de ação previa também a triagem visual, realizando os testes de Snellen nas crianças a partir de cinco anos; Angular "Mãozinha"10, de três a quatro anos; e de Reação à Oclusão, de zero a dois anos. A triagem foi efetuada na creche municipal Centro de Educação Municipal Escola Infantil Maria Aparecida Cacciolato, localizada no bairro Santa Mônica, em Campinas**.

\section{RESULTADOS E DISCUSSÃO}

O segundo questionário aplicado aos usuários do Centro de Saúde comprovou a necessidade de realizar ações de promoção e prevenção da saúde ocular na comunidade do Jardim Santa Mônica.

Em primeiro lugar, observou-se que 58\% dos indivíduos pesquisados referiu acreditar que enxergava de maneira satisfatória, respondendo a questão “O(a) Sr(a). enxerga bem?”. Isto "pode sugerir uma redução da percepção crítica da população sobre sua saúde, tanto pelos formatos das respostas (freqüentemente iniciadas por "eu acho que"), quanto pela pouca importância que os indivíduos parecem dar à saúde ocular, mostrando-se acomodados mesmo quando sua visão está abaixo do ideal.

Além disso, considerando-se que os testes de acuidade visual constituem ações básicas de saúde e, portanto, são prerrogativas das unidades básicas (segundo os princípios do SUS), foi possível notar que essas ações básicas não estão sendo cumpridas de forma satisfatória, visto que apenas $17,6 \%$ dos entrevistados referem ter passado por testes visuais no Cen-

\footnotetext{
* Dr. Cláudio Muniz, médico oftalmologista do Centro de Especialidades de Campinas.

** Professora responsável pelas atividades do projeto na creche Maria Aparecida.
}

tro de Saúde local. Ao mesmo tempo, 58,8\% dos entrevistados referem já ter realizado consultas em serviços de Oftalmologia, encaminhados, porém, em sua maioria, por afecções diversas ou necessidades burocráticas - como, por exemplo, Carteira Nacional de Habilitação -, não diretamente relacionadas à verificação de acuidade visual, e muitos procuraram o serviço privado.

Esses dados revelam um quadro em que a saúde ocular é parcialmente negligenciada. Tal situação pode partir tanto dos usuários, que desconhecem a importância da saúde ocular e os recursos de que dispõem para exercer seus direitos (ações básicas de saúde), ou então das instituições e pessoas, que não realizam efetivamente seu papel de promover a saúde ocular e prevenir problemas visuais.

Observou-se, ainda, que 25 dos 51 entrevistados afirmaram não ter consciência de quais são os cuidados que se deve ter em relação aos olhos, evidenciando ainda mais um quadro de deficiência inclusive de ações educativas para a população. Entre os que puderam citar algumas medidas importantes para garantir a saúde ocular, os principais cuidados mencionados foram: não ficar próximo à televisão ou computador, não ferir os olhos e sempre lavá-los. Apenas um indivíduo citou o uso de óculos, e somente quatro mencionaram a consulta médica, um dado que pode salientar o desconhecimento das pessoas tanto em relação à própria importância dessas duas medidas, quanto à necessidade e direito que possuem de receber medidas de promoção da saúde ocular e prevenção por meio de testes periódicos e tratamentos que possam ser necessários.

O panorama se completa com a informação de que $72,5 \%$ dos usuários pesquisados referiram não usar óculos, e o restante $(27,5 \%)$ afirmou utilizar ou já haver utilizado tal tratamento. Foi possível observar que parte desses indivíduos passou pela última consulta havia mais de um ano, não priorizando a importância de realizar consultas médicas periodicamente. Alguns usuários, ainda, referiram a falta de recursos financeiros como motivo para não utilizarem os óculos, mesmo com recomendação médica, o que mostra a importância da ação do SUS, que deveria promover o acesso de todos às medidas de tratamento.

A partir da aplicação dos questionários à população dos bairros Jardim Santa Mônica e Jardim São Marcos do município de Campinas, São Paulo, pelos estudantes do primeiro ano de Medicina da Unicamp, a análise dos dados revelou:

- Falta de informação quanto às doenças relacionadas ao sistema visual humano;

- Desconhecimento dos direitos dos usuários do SUS, em especial no que diz respeito à saúde ocular; 
- Desconhecimento das formas de prevenção de problemas visuais e cuidados com os olhos;

- Falta de recursos para tratamentos oftalmológicos, gerando casos não tratados ou terapêuticas abandonadas;

- Falta de uma política pública que contemple as estratégias de promoção da saúde ocular e prevenção de problemas visuais.

A partir da definição das necessidades, desenvolveu-se um projeto de ação para amenizar as deficiências encontradas e instrumentalizar a população para que, com atores sociais atuantes no processo de saúde-doença local, pudessem agir como multiplicadores de conhecimento, buscando a sustentabilidade das ações desencadeadas. Esse projeto teve como alvo definido as crianças menores de sete anos. Justifica-se essa escolha devido ao período crítico de plasticidade neural do sistema visual terminar por volta dessa idade, permitindo corrigir totalmente problemas visuais.

O envolvimento de vários atores sociais foi importante na conjugação de esforços nas áreas de saúde e educação, elevando sua abrangência, desencadeando um processo de conscientização e permitindo a continuidade do projeto.

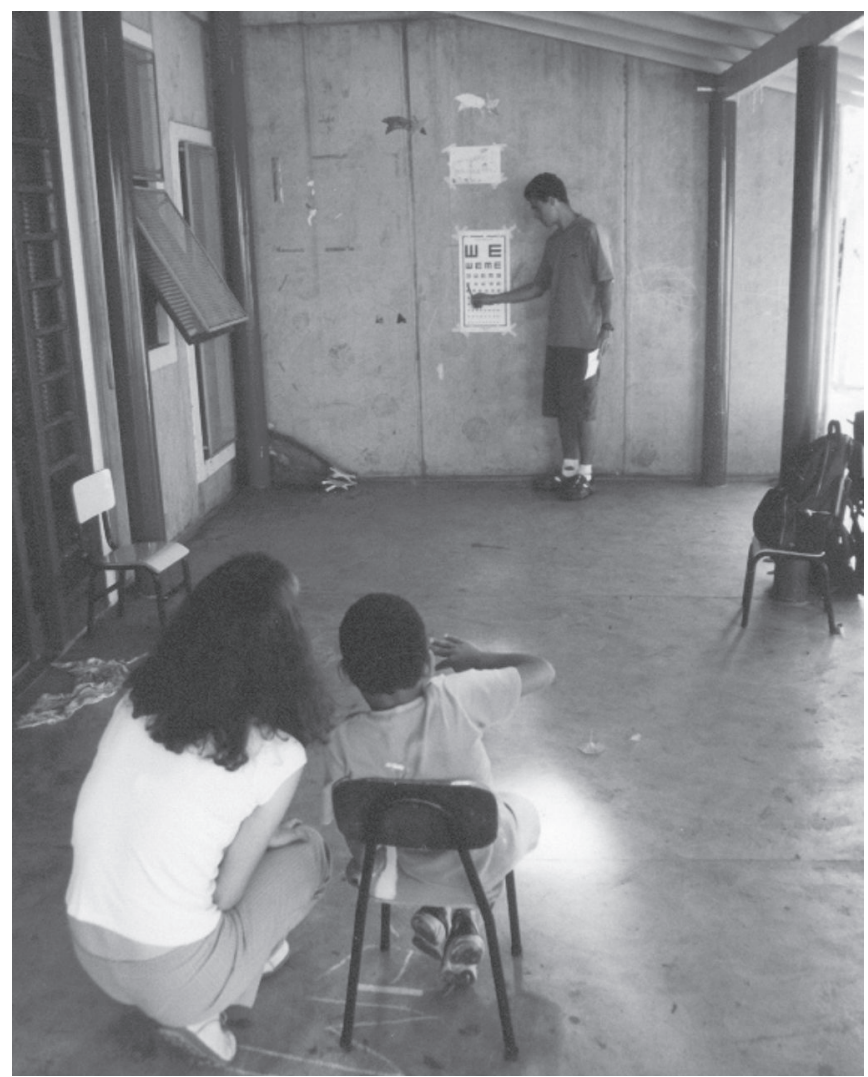

Alunos do primeiro ano (2003) de Medicina realizam testes visuais em crianças da creche em Campinas (SP)
Ao todo, 93 crianças foram triadas e submetidas a testes de acuidade visual - 10 na faixa etária de até 3 anos; 21 de 3 a 4 anos; e 62 entre 5 e 7 anos. Do total de crianças triadas, 80 não apresentaram sinais de deficiência visual; daquelas que apresentaram problemas, 8 foram encaminhadas.

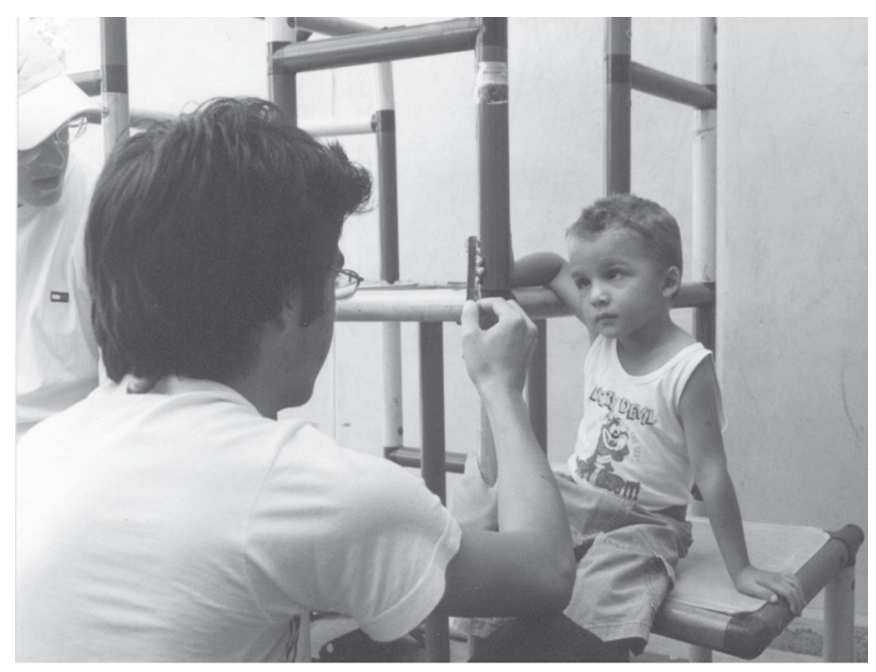

Foi necessário o conhecimento de técnicas básicas de abordagem com algumas crianças, para aprender sobre elas, devido à pouca idade de algumas, ou para lidar com a aversão a alguns testes. A utilização de brinquedos e técnicas simples foi de grande valia para conquistar a atenção da criança ao teste.

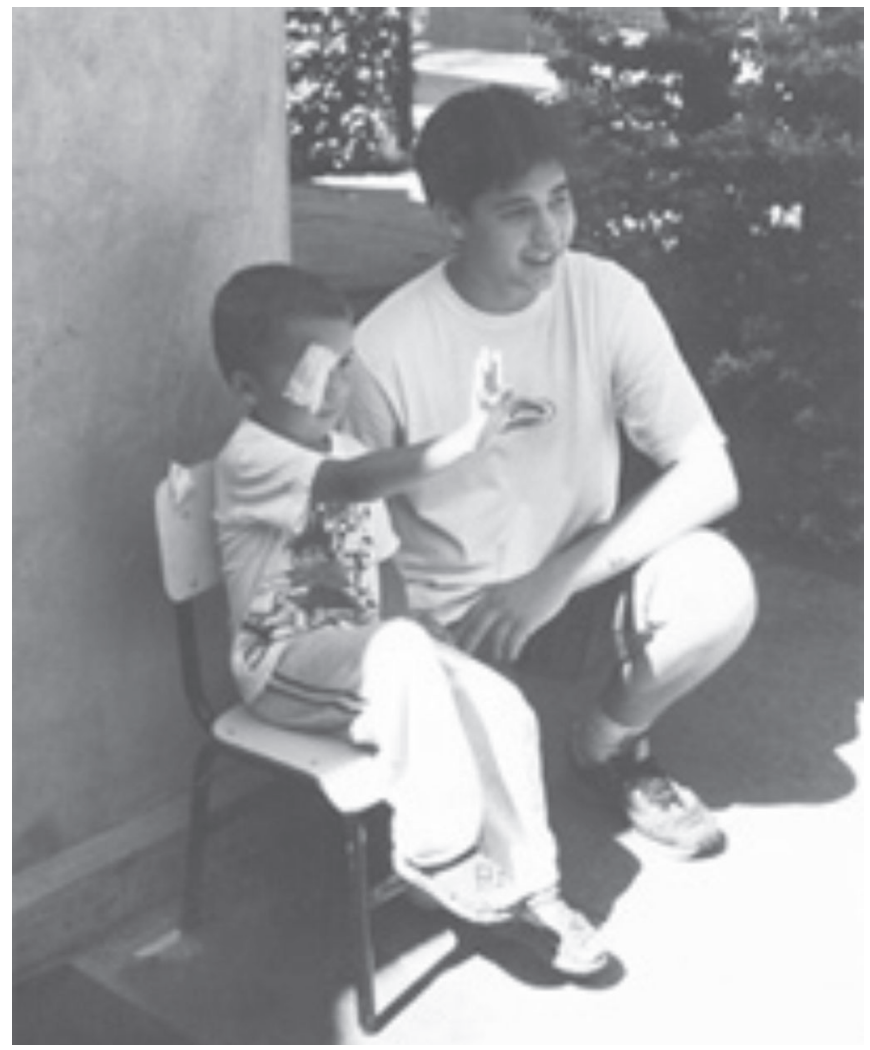


Ao final do projeto, e respeitando o princípio de integralidade do SUS e a responsabilidade inerente ao desencadeamento de ações de saúde numa comunidade, foram fornecidos óculos para os casos confirmados na consulta com oftalmologista. Esta consulta foi agendada pelos professores e pelos profissionais do Centro de Saúde, com vagas reservadas a partir de um acordo estabelecido antes do início do projeto.

Construiu-se, então, um banco de óculos, sustentado inicialmente por doações de armações pela população, pelos estudantes envolvidos no projeto e pela Tecnol, empresa do setor. Esse banco ficou sediado no Centro de Saúde; a confecção das lentes ficou sob a responsabilidade da Óptica da Universidade Estadual de Campinas. Esta ação mostrou-se necessária devido à declaração de alguns usuários, que afirmaram não utilizar óculos devido à falta de recursos para adquiri-los, buscando-se, assim, alternativas para a construção da assistência integral à saúde, papel que deveria ser do SUS.

\section{RECOMENDAÇÕES}

É importante lembrar que o desenvolvimento ocular ocorre nos primeiros sete anos de vida ${ }^{16}$ e, portanto, o setor público/administrativo, a saúde, a educação e a população devem se responsabilizar pelas ações de promoção e proteção à saúde ocular, principalmente desta faixa etária7.

Lembre-se, ainda, que a Lei Federal 8.080/90 pontua que é dever do Estado garantir a saúde por meio da formulação e execução de políticas econômicas e sociais que visem à redução de riscos de doenças e de outros agravos e ao estabelecimento de condições que assegurem acesso universal e igualitário às ações e aos serviços para a sua promoção, proteção e recuperação (Brasil, Lei 8.080/90) ${ }^{14}$.

É importante trabalhar na construção de projetos coletivos, para obtermos respostas mais qualitativas às estratégias do Sistema Único de Saúde e articulá-las com a utilização do recurso pedagógico de práticas de ensino que envolvem universidade, Centro de Saúde e população, formando uma triangulação em que a maioria de seus representantes - alunos, professores, profissionais da saúde e cidadãos - deve se envolver para alcançarmos o SUS que queremos, com direitos e deveres.

\section{CONCLUSÃO}

O trabalho demonstrou a carência de informações de parte da população do bairro de Santa Mônica sobre direitos concernentes à saúde ocular, bem como sobre o acesso ao aprendizado de medidas preventivas contra doenças visuais. Grande parte dela considera satisfatória a condição em que se encontra sua saúde ocular e não intenta averiguar possíveis problemas, mesmo que alguns sinais ou sintomas estejam em evidência.
Tal realidade se torna ainda mais perigosa principalmente quando se trata da saúde ocular de crianças de até sete anos, que está sendo colocada numa escala menor de importância pelos pais, professores e serviço de saúde, visto que exames de acuidade visual são realizados raramente, mesmo na rede pública.

Analisando os números obtidos, vimos que 8,6\% das crianças apresentaram algum tipo de deficiência visual. Esse número é significativo e comprova a importância das atividades realizadas em termos de respeito à promoção da saúde e do bem-estar das crianças que vivem no bairro Santa Mônica, em Campinas.

Ao iniciarem as atividades práticas, os alunos relataram a importância do contato direto com as crianças e com os professores, podendo-se inferir que muitas delas apresentam dificuldades de aprendizado e na atenção às aulas ou atividades, e têm comportamentos apáticos, tanto em sala de aula como no desenvolvimento de atividades extracurriculares. Devido aos vários problemas de visão constatados, acreditamos em um forte indício de correlação entre esses dois fatos.

Sob a supervisão técnica da professora, aprendemos a aplicar diversos tipos de testes de acuidade visual, aprofundamos nossos conhecimentos sobre patologias oculares e pudemos ter contato próximo com a rede pública de saúde, por meio de atividades realizadas no Centro de Saúde do bairro. Constatamos que esse bairro necessitava de investimento nos recursos humanos existentes, visando à promoção da saúde.

Destaque-se o trabalho dos professores e funcionários da creche municipal, de grande importância para realizar os testes de maneira mais organizada e completa, e o conhecimento fluir na prática, demonstrando a importância da construção da parceria, da intersetorialidade e da multiplicação de conhecimentos básicos.

Enquanto proposta de ensino para aprender as ações básicas de saúde e funcionamento do SUS, houve grande aceitação e envolvimento dos alunos, pois puderam acompanhar o levantamento das necessidades de uma amostra da população usuária da unidade de saúde Santa Mônica; a elaboração do projeto junto com a unidade escolar e da saúde; a implantação do projeto; o levantamento dos recursos humanos e materiais necessários; e a organização do banco de óculos para suprir essa necessidade.

Foi constatado que o SUS tem dificuldade de resolver uma questão fundamental: como trabalhar a complexidade de agir e construir políticas públicas que atendam os princípios da integralidade e da eqüidade - promover, proteger e recuperar a saúde -, cumprindo os deveres constitucionais.

Acredita-se que, para os alunos, o aprendizado maior foi na direção de fortalecer a idéia de realizar projetos articulados, em que atuem diferentes instituições e pessoas, com pa- 
péis e interesses definidos claramente para alcançar os objetivos comuns e colaborar na melhoria da qualidade de vida.

A participação neste projeto permitiu aos estudantes experimentar uma nova forma de aprendizado, na qual o conhecimento é construído de forma ativa e horizontal, por meio da gestão conjunta do trabalho com docentes, funcionários da creche e agentes comunitários, desde o levantamento das necessidades locais, até o fornecimento do tratamento aos pacientes triados, vivenciando o princípio de integralidade do SUS.

Foi criado, ainda, um espaço para a elaboração de críticas sobre as políticas de saúde, por meio da percepção da carência na área da saúde ocular, tanto pela ausência de conhecimento da população sobre seus direitos, quanto pela pouca importância dada pelos profissionais de saúde visual.

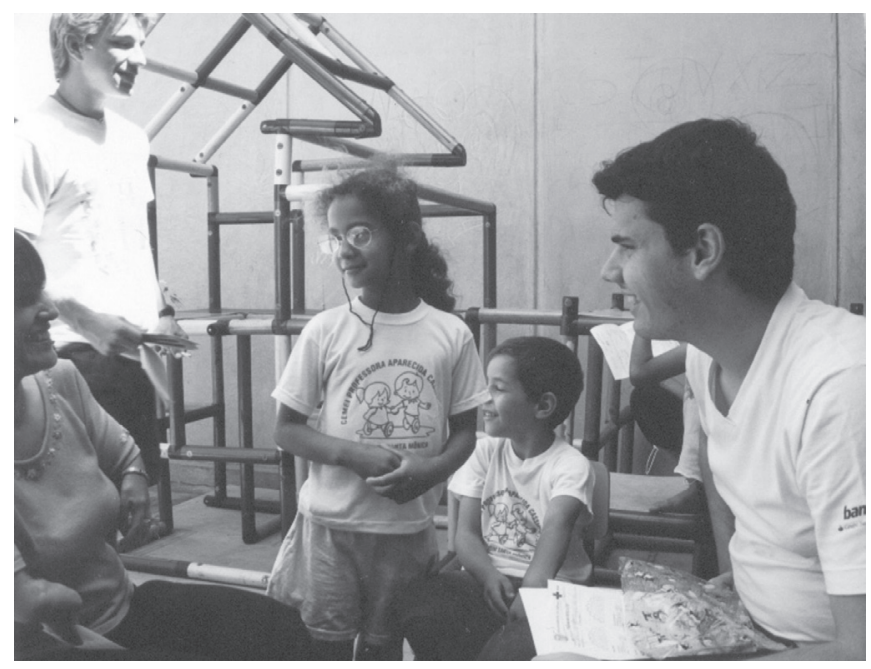

Agradecimentos

A todos aqueles que colaboraram para que este trabalho se realizasse, fábrica de armações de óculos Tecnol (Campinas), agentes comunitários do Centro de Saúde Santa Mônica, professores e auxiliares da Creche Municipal Centro de Educação Municipal Escola Infantil - Professora Aparecida Cassiolato.

\section{REFERÊNCIAS}

1. Brasil. Constituição da República do Brasil. Rio de Janeiro: Degrau Cultural; 1988. Arto 196, Art 30 (al. VII).

2. Sperandio, Ana Maria Girotti. (org). O Processo de construção da Rede de Municípios Potencialmente Saudáveis, Vol. II, Campinas, IPES Editorial; 2004.

3. OPAS. Organização Pan-Americana da Saúde, Divisão de Promoção e Proteção da Saúde. Municípios e Comunidades saudáveis. Guia para prefeitos para promover qualidade de vida. Brasília: OPAS/ W.K. Kellogg Foundation; 2003.
4. Brasil. Ministério da Saúde. Política Nacional de Promoção da Saúde. Documento Preliminar. Editora Ministério da Saúde: Brasília; 2006.

5. Padilha R. Q. , Feuerwerker L. C. M. As Políticas Públicas e a Formação de Médicos. Rev Bras Educ Méd. 2002; 26(2): 3-4.

6. Martins, MA. Ensino Médico no Brasil. Aula introdutória do Módulo de Ações de Saúde Pública do Primeiro Ano de Medicina de 2006 da FCM. São Paulo: FCM/ UNICAMP;2006. [ Power-Point 16/3/2006].

7. Sperandio AMG. A promoção da saúde ocular e a prevenção precoce de problemas visuais nos serviços de saúde pública de Campinas. Rev Saúde Pública, 1999; 33:513-520.

8. Milton RA, Kara-José, N. Manual de instruções. Conselho Brasileiro de Oftalmologia. Campanha Nacional de Prevenção da Cegueira e Reabilitação Visual,[Salvador]; 1998.

9. Kara-José N, Oliveira RC. Mitos e verdades: olhos. São Paulo: Contexto;1997.

10. Lin-Fu JS. Vision screening of children. US Department of Health. Maternal and Child Health Service; 1971. p. 12-13.

11. Thylefors B. A simplified methodology for the assessment of blindness and its main causes. World Health Stat Q, 1987; 40: 129-41.

12. Barry JC, König HH. Test characteristics of orthoptic screening examination in 3 years old kindegarten children. British Journal of Ophthalmology 2003; 87: 909-916.

13. Thiollent, M. Metodologia da Pesquisa-ação. $8^{\mathbf{a}}$ ed. São Paulo: Cortez; 1998.

14. Brasil. Lei 8080. Lei Orgânica da Saúde. Diário Oficial da União, Brasília,19 de Setembro de 1990. Disponível em:http://www.saude.gov.br/doc/lei8080.htm.

15. Couto Jr., AS. Considerações preliminares sobre a identificação das deficiências visuais em pré-escolares e escolares. Monografia de conclusão do Curso de Pós-Graduação em Oftalmologia Clínica e Cirúrgica. Rio de Janeiro: Instituto Benjamin Constant; 1992.

16. Crawford JS, Morin JD. The eye in childhood. Canadá: Departament of Ophthalmology / Hospital for Sick Children; 1982.

\section{Conflito de Interesse}

Declarou não haver.

\section{Endereço para correspondência}

Ana Maria Girotti Sperandio

Rua Emílio Romanetti, 246

13278-352 - Valinhos - SP

e-mail: amgspera@uol.com.br 
ANEXO

\section{Departamento de Medicina Preventiva \\ Faculdade de Ciências Médica \\ UNICAMP}

Centro de Saúde Santa Mônica

Prefeitura Municipal de Campinas

\section{FORMULÁRIO}

Nome:

Idade:___ Número de matrícula no CS:

1. O Sr(a) acha que enxerga bem?

2. O Sr(a) já fez um teste de visão no centro de saúde? Quando? Quem fez?

3. O Sr(a) passou alguma vez no serviço de oftalmologia, no médico de olhos?

4. Teve dificuldade para marcar a consulta?

5. $\operatorname{OSr}(\mathrm{a})$ faz algum tipo de tratamento visual? Qual?

6. $\mathrm{OSr}(\mathrm{a})$ usa óculos? Desde que idade? Quando trocou as últimas lentes?

7. O Sr(a) sabe quais são os cuidados que devemos ter com os nossos olhos? 
O nome correto do autor do artigo intitulado "A Universidade colaborando na construção de um projeto de promoção da saúde: relato de experiência de um grupo de alunos de Medicina da Unicamp, Campinas, SP, Brasil", publicado na Rev. Bras. Educ Med. 2006; 30(3), é MARCOS THEÓPHILO GALASSO. 
O arquivo disponível sofreu correções conforme ERRATA publicada no Volume 31 Número 3 da revista. 